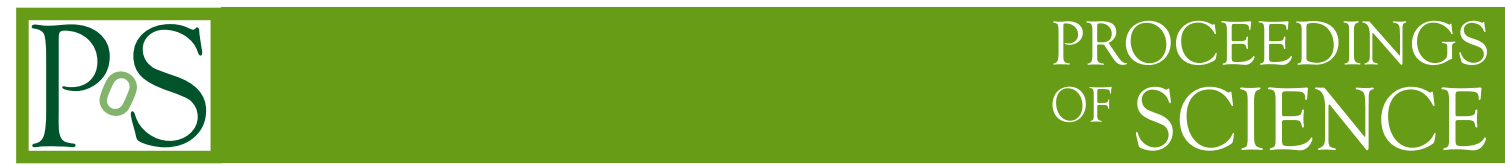

\title{
Search for RPV Scalar Leptons at Tevatron
}

\section{Shan-Huei Chuang ${ }^{* \dagger}$}

University of Wisconsin - Madison, USA

E-mail: schuangefnal.gov

We reviewed CDF and D $\varnothing$ searches for $R$-parity violation supersymmetry in leptons involved final states using up to $344 \pm 21 \mathrm{pb}^{-1}$ Tevatron Run II data of $p \bar{p}$ collisions at $\sqrt{s}=1.96 \mathrm{TeV}$. All the results were in good agreement with the Standard Model expectations. No evidence of new physics was observed. However, owing to the improvement on detectors, energy and luminosity from Run I to Run II, the limits for the existence of $R$-parity violation supersymmetry have been greatly advanced.

International Europhysics Conference on High Energy Physics

Lisboa, Portugal

July 21st - 27th 2005

${ }^{*}$ Speaker.

†On Behalf of the CDF and D $\varnothing$ Collaborations 


\section{Introduction}

Although the Standard Model (SM) has been remarkably successful in describing presently known phenomena there are arguments to consider it as a low-energy effective, hence the construction of its extensions to high energies. Supersymmetry (SUSY) is the most appealing SM extension. Mainly motivated by the cancellation of quadratic divergences in scalar boson mass loop corrections, SUSY postulates such a symmetry that every fermion has a bosonic superpartner and every boson has a fermionic superpartner [1]. The $R$ parity is defined as $R \equiv(-1)^{3 B+L+2 J}$. $R= \pm 1$ for ${ }_{\text {most SUSY models }}^{\text {the Stand }}$ SUSY by itself does not impose $R$ parity conservation. In a minimal $R$ parity violation $(\not R)$ SUSY model the gauge invariance of the minimal supersymmetric extension of Standard Model (MSSM) allows the following additional Yukawa couplings in the Lagrangian:

$$
\mathscr{L}_{\not R} \equiv\left[\lambda_{i j k} L_{i} L_{j} \bar{E}_{k}+\lambda_{i j k}^{\prime} L_{i} Q_{j} \bar{D}_{k}+\lambda_{i j k}^{\prime \prime} \bar{D}_{i} \bar{D}_{j} \bar{U}_{k}\right]
$$

where $i, j, k$ are family indices [2]. The $\lambda$ and $\lambda^{\prime}$ terms violate lepton number (LNV) and the $\lambda^{\prime \prime}$ terms violate baryon number (BNV). Proton decay via squark $\tilde{d}_{2}$ forbids the co-existence of LNV and BNV but not either one. In analogy to the standard Yukawa couplings one expects a hierarchical structure among the $\not R$ couplings and hence the lightest superparticle (LSP) to decay dominantly into one of the $45 \not R$ channels.

\section{Search for $R$-parity Violation Decay of Sneutrino}

CDF searched in $344 \pm 21 \mathrm{pb}^{-1}$ Run II data for high mass resonance decaying into an electron plus a muon and interpreted the results in the context of $\not \lambda \lambda_{311}^{\prime}$ production and $\lambda_{132}$ decay of $d \bar{d} \rightarrow$ $\tilde{v}_{\tau} \rightarrow e \mu$ [3]. The high $p_{t} e \mu$ signature let in little SM backgrounds. The search used high $p_{t}$ lepton



Figure 1: The mass spectrum of data observation vs SM expectation, showing good agreement. The $\tilde{v}_{\tau}$ signal contribution is hypothetical. triggers and selected events with $N_{e} \geq 1$ and $N_{\mu} \geq$ 1 , requiring each lepton to have $p_{t}>20 \mathrm{GeV}$ and be isolated and each lepton pair to be close at beam axis with $\left|\Delta z_{0}\right|<5 \mathrm{~cm}$ and have opposite signs of charge. Signal and SM backgrounds (except QCD) were modeled using PYTHIA (jet data) with the $h_{S M}^{0}$ particle being a functional substitute for $\tilde{v}_{\tau}$. See Figure 1. Uncertainties on acceptance included $9.2 \%$ due to MC generator, $3.2 \%$ due to momentum resolution and $2.4 \%$ due to parton distribution function; on backgrounds the largest was the $16 \%$ from QCD. Observing no excess in data $95 \%$ CL limits of $\tilde{v}_{\tau}$ production cross-section as well as of $\lambda_{311}^{\prime}$ and of $\lambda_{132}$ were set. See Figure 2 .

\section{Search for $\not X$ Production and Decay of Slepton via the $L Q \bar{D}$ Coupling $\lambda_{211}^{\prime}$}

$\mathrm{D} \varnothing$ searched in $154 \pm 10 \mathrm{pb}^{-1}$ Run II data for the resonant production of $\tilde{\mu}$ which leads to a final state with two muons and two jets and the advantage of all the masses reconstructable, 

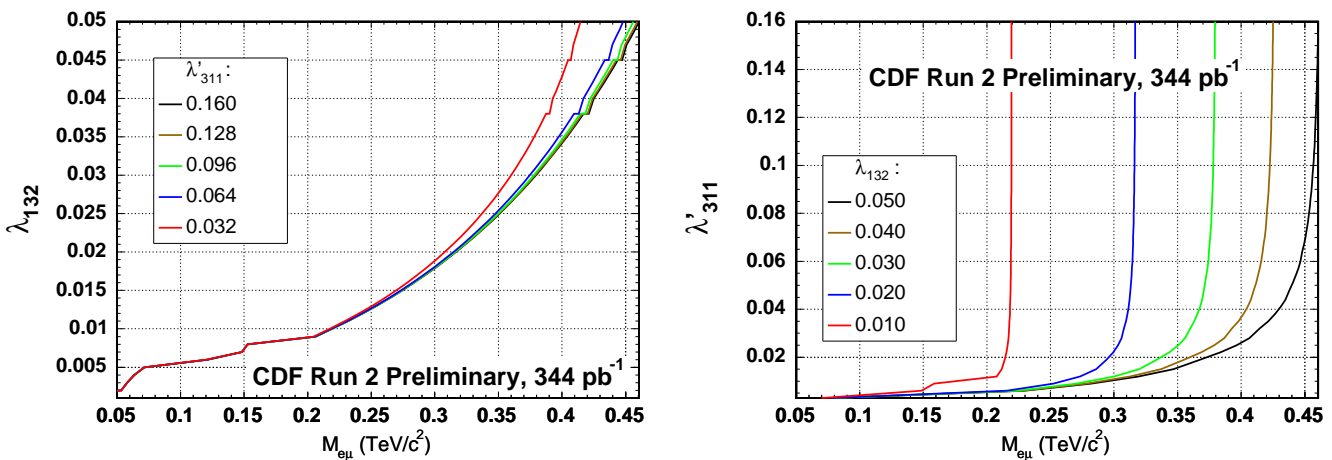

Figure 2: The upper limits of $\lambda_{132}$ for fixed $\lambda_{311}^{\prime}$ (left) and of $\lambda_{311}^{\prime}$ for fixed $\lambda_{132}$ (right) as a function of $m_{\tilde{v}_{\tau}}$.

Figure 3 (left), assuming $\lambda_{211}^{\prime}$ is the dominant $\not 1$ coupling [4]. The search used dimuon triggers and selected events with $\mathrm{S} / \sqrt{\mathrm{B}}$-optimized mass dependent cuts: $\not_{t}<60 \mathrm{GeV}, 2$ isolated muons with $p_{t}{ }^{\left\{\mu_{1}, \mu_{2}\right\}}>\left\{21.25+0.1875\left(m_{\tilde{\mu}}-m_{\tilde{\chi}_{1}^{0}}\right), 10\right\} \mathrm{GeV}$ and $p_{t}{ }^{\mu_{1}}+p_{t}{ }^{\mu_{2}}>60 \mathrm{GeV}, 2$ jets in $|\eta|<2$ and $E_{t}\left\{j_{1}, j_{2}\right\}>\{25,15\} \mathrm{GeV}, m_{\tilde{\chi}_{1}^{0}}^{\mathrm{MC}}-40<m_{\tilde{\chi}_{1}^{0}}<m_{\tilde{\chi}_{1}^{0}}^{\mathrm{MC}}+20 \mathrm{GeV},\left|m_{\tilde{\mu}}^{\mathrm{MC}}-m_{\tilde{\mu}}\right|<0.2 m_{\tilde{\mu}}^{\mathrm{MC}}$, rejecting $Z$ with $\left|m_{\mu \mu}-91\right|<m_{\tilde{\chi}_{1}^{0}} / 9+5 \mathrm{GeV}$ and suppressing QCD by requiring large angle between any two final-state objects. Signal was modeled with SUSYGEN, scanning over $25\left\{m_{\tilde{\mu}}, m_{\tilde{\chi}_{1}^{0}}\right\}$ points with $\lambda_{211}^{\prime}=0.07, A_{0}=0, \tan \beta=2$ and the higgsino mass mixing parameter $\mu<0$. The only nonnegligible background $Z+2 j$ was modeled with PYTHIA. By far the largest uncertainty $\sim 30 \%$ was due to jet energy scale. Reasonable agreement was found for all points, e.g. expected : observed $=1.1 \pm 0.4: 2$ events. Observing no excess in data 95\% CL limits of $\tilde{\mu}$ production cross-section and of $\lambda_{211}^{\prime}$ were set, as shown in Figure 3 (middle and right).
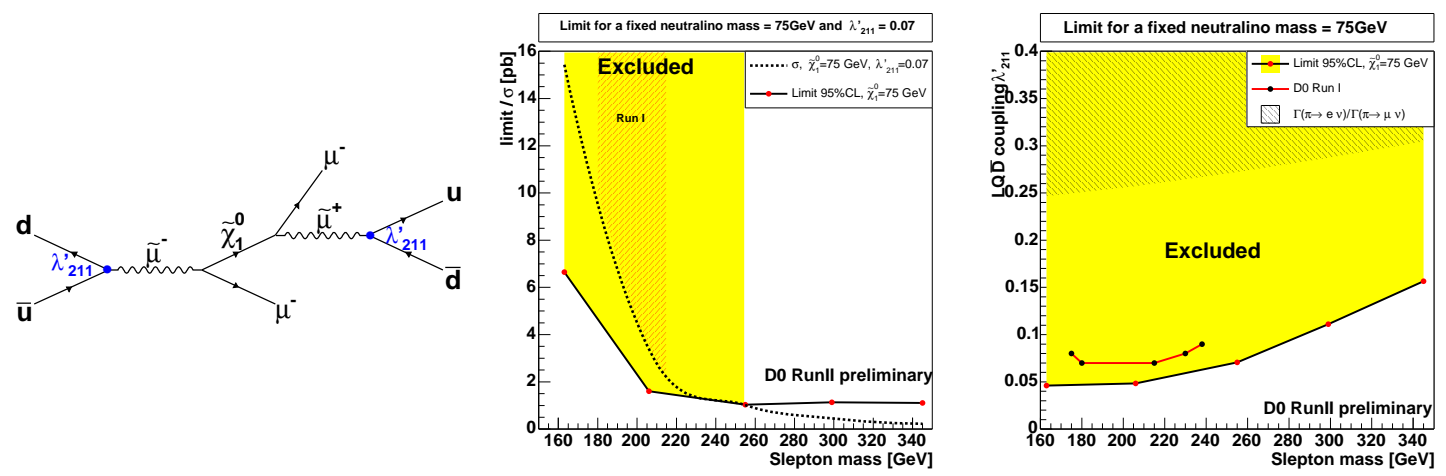

Figure 3: Feynman diagram for $\not R \tilde{\mu}$ production and leptonic $\tilde{\chi}_{1}^{0}$ decay (hadronic not drawn) via $\lambda_{211}^{\prime}$. The $\mu$ or quark directly from the $\tilde{\chi}_{1}^{0}$ decay is expected to have low $p_{t}$ since its sparticle sister is highly virtual (left). The $\tilde{\mu}$ production cross-section limits (middle) and the $\lambda_{211}^{\prime}$ limits (right) as a function of $m_{\tilde{\mu}}$.

\section{Search for RPV Decay of Chargino/Neutralino into $\geq 3$ Lepton Final State}

$\mathrm{D} \varnothing$ has searched for $\not \subset$ leptonic decays of $\tilde{\chi}_{1}^{0}$ via $\lambda_{121}, \lambda_{122}, \lambda_{133}$ into final states that contain $e e \ell, \mu \mu \ell, e e \tau_{h}$ where $\ell \in\{e, \mu\}$ separately $[5,6,7]$. The searches commonly considered the sce- 
nario in which, given the $\not R$ couplings have been set significantly smaller than the gauge couplings by low-energy experiments [8], most SUSY processes conserve $R$ parity and only LSP can $\not R$-decay into SM particles. Sparticles can only be pair produced at Tevatron [1]. In the processes of $p \bar{p} \rightarrow$ SP-pair ... $\rightarrow$ LSP-pair ... ${ }^{\not R} \rightarrow \ell \ell v \ell \ell v \ldots$ at least 4 leptons are in final state. Event selections went commonly as follow: isolated high or medium $p_{t}$

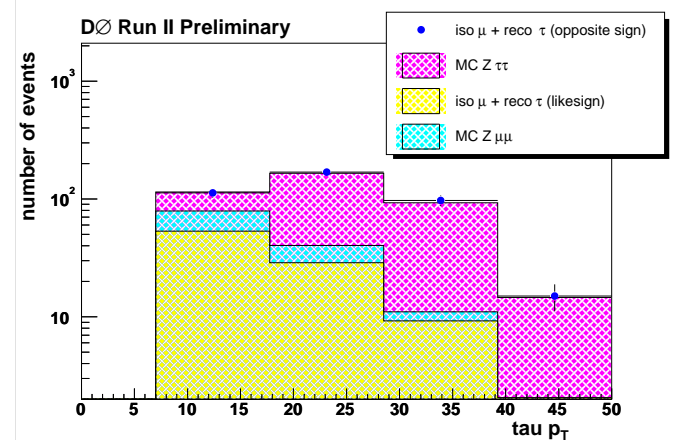

Figure 4: The $p_{t}$ spectrum of hadronically decayed tauon $\left(\tau_{h}\right)$ candidates after neural network identification. dielectron or dimuon with invariant mass outside $\Upsilon$ and $Z$ regions, an additional low $p_{t}$ lepton (see Figure 4), and significant $\not_{t}$ presence to reduce QCD background; the 4th lepton was not required to increase signal acceptances. Signals were modeled with SUSYGEN, scanning over $140<m_{1 / 2}<280$ GeV. SM backgrounds (except QCD), of which the largest was Drell-Yan, were estimated with PYTHIA (jet data). Uncertainties on acceptances were driven by the $6 \%$ on luminosities; on backgrounds $60 \%$ of total were from DY and QCD. Results and related SUSY parameters are summarized in Table 1.

\begin{tabular}{cccccccccc}
\hline \hline & $\mathscr{L}_{\text {int }}\left(\mathrm{pb}^{-1}\right)$ & $\lambda$ constraint & $\tan \beta$ & $m_{0}$ & $N_{\text {expected }}: N_{\text {observed }}$ & $m_{\tilde{\chi}_{1}^{0}}^{\mu<0}$ & $m_{\tilde{\chi}_{1}^{ \pm}}^{\mu<0}$ & $m_{\tilde{\chi}_{1}^{0}}^{\mu>0}$ & $m_{\tilde{\chi}_{1}^{ \pm}}^{\mu>0}$ \\
\hline \hline$e e \ell$ & $238 \pm 16$ & $\lambda_{121}=0.010$ & 5 & 250 & $0.45 \pm 0.43: 0$ & 95 & 181 & 97 & 183 \\
\hline$\mu \mu \ell$ & $160 \pm 10$ & $\lambda_{122}=0.001$ & 5 & 250 & $0.63 \pm 1.93: 2$ & 84 & 160 & 90 & 165 \\
\hline$e e \tau_{h}$ & $199 \pm 13$ & $\lambda_{133}=0.003$ & 10 & 80 & $1.04 \pm 1.42: 0$ & & & 66 & 118 \\
\hline \hline
\end{tabular}

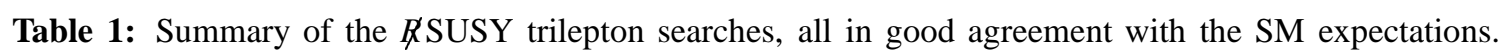
$A_{0}=0$. The $95 \% \mathrm{CL}$ lower $m_{\tilde{\chi}_{1}^{0}}$ or $m_{\tilde{\chi}_{1}^{ \pm}}$limits $(\mathrm{GeV})$ for $\mu>0$ or $\mu<0$ are shown in the last four columns.

\section{Conclusion}

As CDF and D $\varnothing$ have searched so far there is no evidence of $\not R S U S Y$ yet. The limits of $R$-parity violation supersymmetry have been either newly set or pushed way further.

\section{References}

[1] A Supersymmetry Primer, Stephen P. Martin, hep-ph/9709356, April 71999.

[2] S.Dimopoulos \& L.Hall, Phys.Lett.B207:210, 1988. H.Dreiner \& G.Ross, Nucl.Phys.B365:597, 1991.

[3] A Search for RPV Sneutrino Decay to $e \mu$, CDF conference note 7616, 2005.

[4] Search for Resonant Slepton Production via the $L Q \bar{D}$ Coupling $\lambda_{211}^{\prime}$, D0note4535-CONF, 2004.

[5] Search for SUSY with RPV in the eel Final State, D0note4522-CONF, 2004.

[6] Search for SUSY with RPV in the $\mu \mu \ell$ Final State, D0note4490-CONF, 2004.

[7] Search for SUSY with RPV in the ee $\tau$ Final State, D0note4595-CONF, 2005.

[8] V.Barger et al., Phys.Rev.D40:2987’89, Phys.Rev.D50:4299'94. X.Tata et al., Phys.Rev.D51:2180’95. 